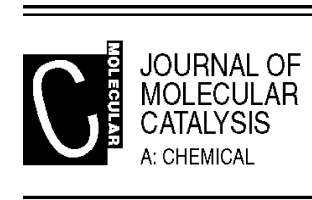

ELSEVIER

Journal of Molecular Catalysis A: Chemical 158 (2000) 301-307

www.elsevier.com/locate/molcata

\title{
Catalytic synthesis of carbon nanostructures from polymer precursors
}

\author{
Nadezhda I. Maksimova a,b ${ }^{\text {a }}$ Oleg P. Krivoruchko ${ }^{\text {a, * }}$, Gerhard Mestl ${ }^{\text {, }}$, \\ Vladimir I. Zaikovskii ${ }^{a}$, Andrey L. Chuvilin ${ }^{a}$, Aleksey N. Salanov ${ }^{\text {a }}$, Elena B. Burgina ${ }^{\text {a }}$ \\ ${ }^{a}$ Boreskov Institute of Catalysis, Prospect Ak. Lavrentieva, 5, 630090, Novosibirsk, Russia \\ ${ }^{\mathrm{b}}$ Fritz-Haber-Institut der Max-Planck-Gesellschaft, Faradayweg 4-6, D-14195, Berlin, Germany
}

\begin{abstract}
Carbon nanostructures were prepared by decomposition of polyethylene and polyvinyl alcohol using an iron catalyst at $600-750^{\circ} \mathrm{C}$ under a nitrogen flow. Heating a uniform distribution of catalyst particles in the polymer bulk to $600^{\circ} \mathrm{C}$ under flowing nitrogen led to the formation of amorphous carbon incorporating uniformly distributed catalyst particles. Subsequent heating of the samples to $750^{\circ} \mathrm{C}$ in a nitrogen flow led to the catalytic graphitization of the amorphous carbon matrix with the formation of different carbon nanostructures, i.e. carbon nanotubes. The mechanism of the formation of the obtained carbon nanostructures is discussed. (C) 2000 Elsevier Science B.V. All rights reserved.
\end{abstract}

Keywords: Polyvinyl alcohol; Polyethylene; Catalytic decomposition; Carbon filaments; Carbon nanotubes

\section{Introduction}

Since the discovery of fullerenes [1] and carbon nanotubes [2], an extensive experimental research has been performed to produce new carbon nanostructures, which are mainly classified into two groups, i.e. nanoparticles and nanotubes. New carbon materials attract particular interest due to a wide variety of their structures [3] and the prediction of their unique properties and potential applications [4]. Increasing attention is paid to carbon nanotubes because their

\footnotetext{
* Corresponding author. Tel.: +007-3832-340-002; fax: +0073832-343-056.

E-mail address: opkriv@ catalysis.nsk.su (O.P. Krivoruchko).
}

properties may possibly be modified by filling or intercalating metals into the nanotubes $[5,6]$. The catalytic decomposition of hydrocarbons is a promising way for the production of carbon nanotubes on large scales [7,8]. Amorphous carbon is formed during the catalytic decomposition of hydrocarbons. The subsequent interaction of carbon with the catalyst leads to graphitization. It is known that amorphous carbon transforms into graphite in a temperature range between $2500^{\circ} \mathrm{C}$ and $3500^{\circ} \mathrm{C}$ [9]. In the presence of $3 \mathrm{~d}$ metals and their alloys, graphitization occurs at lower temperatures (600$900^{\circ} \mathrm{C}$ ) [10]. Previously, it was observed that amorphous carbon transforms into graphite through an interaction with $\mathrm{Fe}, \mathrm{Co}$, or $\mathrm{Ni}$ particles under vacuum in the temperature range between $600^{\circ} \mathrm{C}$ and $900^{\circ} \mathrm{C}$ via the formation of 
liquid metal-carbon particles $[11,12]$. Recently, we investigated the catalytic decomposition of polymers up to $600^{\circ} \mathrm{C}$ [13]. It was discovered that the polymers could be used as carbon precursors. It was suggested that such amorphous carbon undergoes a subsequent graphitization in the presence of catalyst particles in the temperature range between $600^{\circ} \mathrm{C}$ and $900^{\circ} \mathrm{C}$ in an inert gas flow giving carbon structures of dimensions comparable to those of the catalyst particles.

In this paper, we describe the synthesis and the mechanism of the formation of different carbon nanostructures from polyethylene and polyvinyl alcohol carbon precursors in the presence of an iron catalyst. The samples were characterized by transmission electron microscopy (TEM), scanning electron microscopy (SEM), X-ray powder diffraction (XRD), Raman and IR spectroscopies.

\section{Experimental}

The polymers are dissolved in an appropriate solvent (ortho-xylene for polyethylene, and water for polyvinyl alcohol). Iron hydroxide, as the catalyst precursor, was prepared by co-precipitation of $\mathrm{Fe}\left(\mathrm{NO}_{3}\right)_{3} \cdot 9 \mathrm{H}_{2} \mathrm{O}$ and $\mathrm{NaOH}$ solutions at $\mathrm{pH}=12$. The obtained suspension was washed in distilled water to $\mathrm{pH} \cong 6$ by a decantation method, then filtered and dried at $150^{\circ} \mathrm{C}$ until constant weight. The iron hydroxide (getite) samples consist of needle-shaped crystals with characteristic sizes of $l \times h \cong 250 \times 20 \mathrm{~nm}$ as determined by TEM and a specific surface area of ca. $80 \mathrm{~m}^{2} \mathrm{~g}^{-1}$ measured by the BET method. The iron hydroxide was added to the polymer solutions in the weight ratio of 1:20 relative to the dry polymer. The viscous suspension of the catalyst precursor and the polymer is stirred at $70^{\circ} \mathrm{C}$ until uniformity is reached, and then poured onto a smooth glass surface. The residual solvent was removed by heating at $90^{\circ} \mathrm{C}$. The obtained polymer films of ca. $0.3-0.5 \mathrm{~mm}$ thickness are sliced to $2 \times 5 \mathrm{~mm}$ pieces and loaded into a quartz reactor. The samples were heated in the quartz reactor up to $750^{\circ} \mathrm{C}$ with a heating rate of $2.5^{\circ} \mathrm{min}^{-1}$ under a nitrogen flow with a rate of $50030 \mathrm{ml} / \mathrm{min}$ with holding periods for $2 \mathrm{~h}$ at $250^{\circ} \mathrm{C}, 400^{\circ} \mathrm{C}, 600^{\circ} \mathrm{C}$, and $750^{\circ} \mathrm{C}$, respectively, to remove the volatile products of the polymer decomposition, and the subsequent carbonization and graphitization of the samples. During this procedure, $\alpha-\mathrm{FeO}(\mathrm{OH})$ is transformed into iron oxides and then reduced to the metal.

The TEM and HRTEM images were obtained with JEM100CX and JEM 2010 microscopes, respectively. SEM was performed with a TESLA-BS-350. IR spectroscopy measurements were performed with a LabRam spectrometer (Dilor) using a 632-nm excitation at $25 \mathrm{~mW}$ laser power. The spectral resolution of $2.5 \mathrm{~cm}^{-1}$. XRD patterns were recorded on a Siemens URD-63 diffractometer using $\mathrm{CuK} \alpha$ radiation and a graphite crystal monochromator.

In the following, the samples are abbreviated as shown in Table 1.

\section{Results and discussion}

The total yield of gaseous and liquid hydrocarbons from the thermal decomposition of polyethylene is about 98\% [14], and, consequently, the amount of solid product is very small. In our previous studies [13,15], it was shown that the decomposition of polyethylene without catalyst (the samples PE1 and PE2) leads to the formation of an amorphous carbon.

Table 1

\begin{tabular}{lllll}
\hline Sample decomposition & Polyethylene & Polyvinyl alcohol & $\begin{array}{l}\text { Polyethylene } \\
\text { with catalyst }\end{array}$ & $\begin{array}{l}\text { Polyvinyl alcohol } \\
\text { with catalyst }\end{array}$ \\
\hline$T=600^{\circ} \mathrm{C}$ & PE1 & PVA1 & PE-Fe1 & PVA-Fe1 \\
$T=750^{\circ} \mathrm{C}$ & PE2 & PVA2 & PE-Fe2 & PVA-Fe2 \\
\hline
\end{tabular}




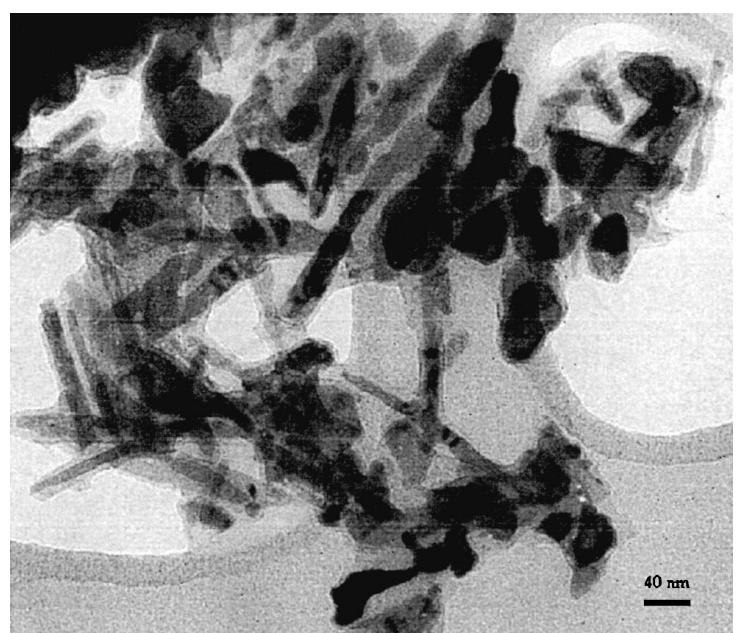

Fig. 1. The TEM image of the polyethylene incorporating catalyst particles heated to $600^{\circ} \mathrm{C}$ in a nitrogen flow.

In the samples PE-Fe1 and PE-Fe2, the yield of amorphous carbon has increased relatively to the amorphous carbon yield of the PE1 and PE2 samples. According to XRD data (not shown), the sample PE-Fe1 contains iron oxides and amorphous carbon and the sample PE-Fe2 contains iron metal and amorphous carbon. The absence of graphite reflections for the sample
PE-Fe2 may possibly be the result of disorientation of graphite layers (turbostratic graphite).

The TEM image shown in Fig. 1 of the sample PE-Fe1 demonstrates the formation of amorphous carbon around elongated catalyst particles. The composition of the sample has been dramatically changed after heating to $750^{\circ} \mathrm{C}$ (Fig. 2). In the sample PE-Fe2, most of the elongated catalyst particles covered by amorphous carbon have disappeared. It is interesting to note that large, spherical catalyst particles were detected that were not covered by carbon. The predominant carbon structures found after this treatment were hollow carbon tubules assembled in large agglomerates. The observed formation of two types of catalyst particles and two types of carbon structures might result from two different processes which occur between $600^{\circ} \mathrm{C}$ and $750^{\circ} \mathrm{C}$. In the first process, amorphous carbon may undergo partial graphitization, thus, forming shells around the catalyst particles. In the second process, most of the metal particles leave their carbon shells and accumulate in large spherical aggregates. As a result of these processes, hollow carbon tubules were mostly formed with a shape replicating the

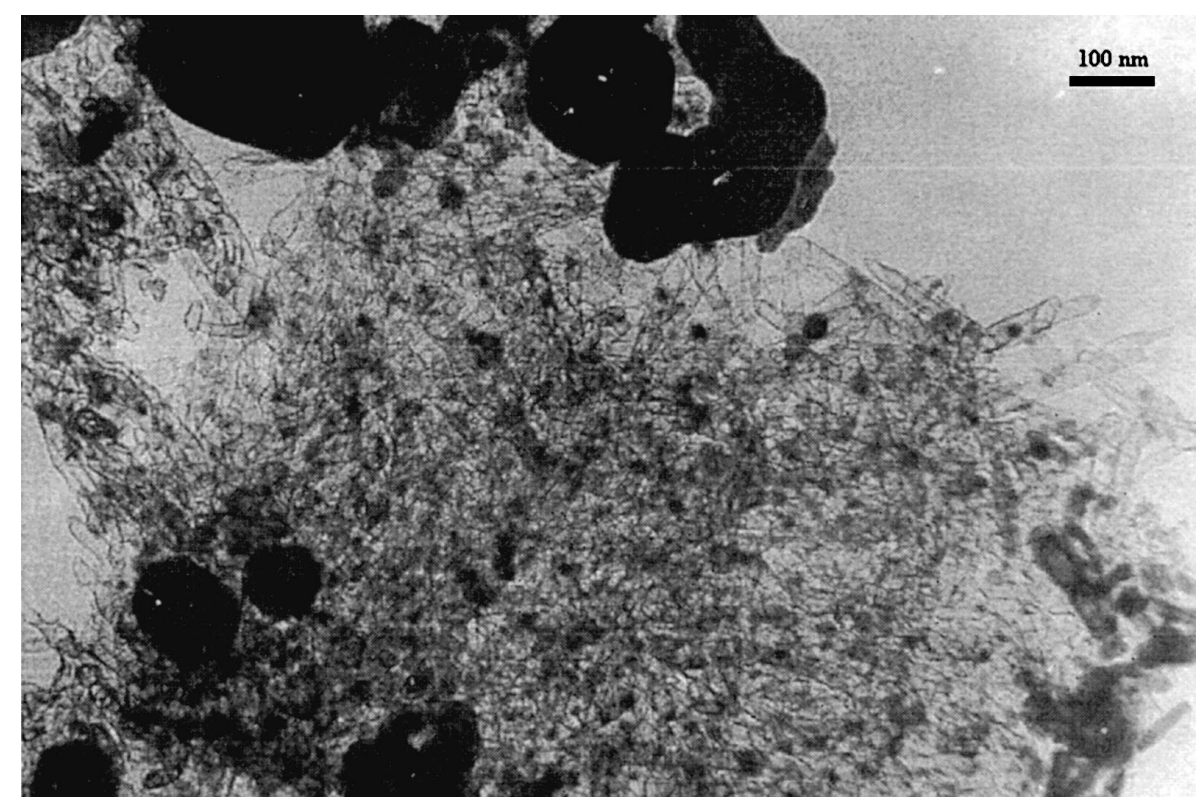

Fig. 2. The TEM image of the polyethylene incorporating catalyst particles heated to $750^{\circ} \mathrm{C}$ in a nitrogen flow. 


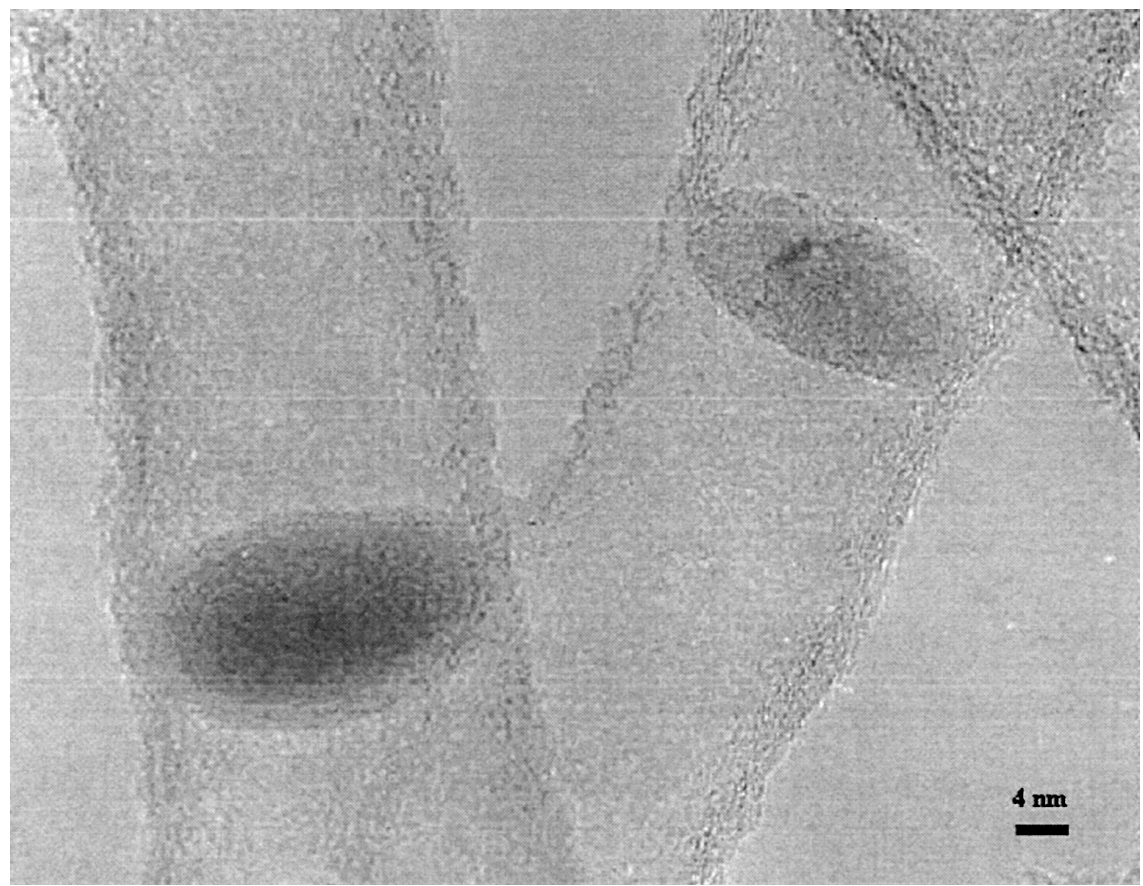

Fig. 3. The HRTEM image of the fragment of carbon tubule containing oblate catalyst particle in the inner cavity.

former surface of the catalyst particles. The mean dimensions of the carbon tubules (width - $20 \mathrm{~nm}$, length - $300 \mathrm{~nm}$ ) corresponded to the size of the amorphous carbon particles formed at $600^{\circ} \mathrm{C}$ around the catalyst particles. According to the HRTEM data (Fig. 3), the walls of the carbon tubules were formed from disordered graphitic layers oriented along the tubule axis (Fig. 3). In Fig. 3, small oblate catalyst particles are seen which remained in the tubule cavities and are covered by carbon.

It is interesting to note that the formation of the hollow tubules occurred via the movement of the Fe particles through defects in the carbon cover. The movement of the metal particles very likely proceeds via their transition into a liquid-like state $[11,12,16,17]$. We believe that liquid-like metal particles are formed via a solid-solid interaction of the metal with the amorphous carbon in the temperature range between $600^{\circ} \mathrm{C}$ and $750^{\circ} \mathrm{C}$. The liquid-like catalyst particles only exists during the reaction and their nature remains a matter of discussion. Obviously, the observed large metal particles were formed by the merger of small metal particles released from their carbon covers.

The TEM image (Fig. 4) of the sample PE$\mathrm{Fe} 2$ after purification in acid solutions (aqueous

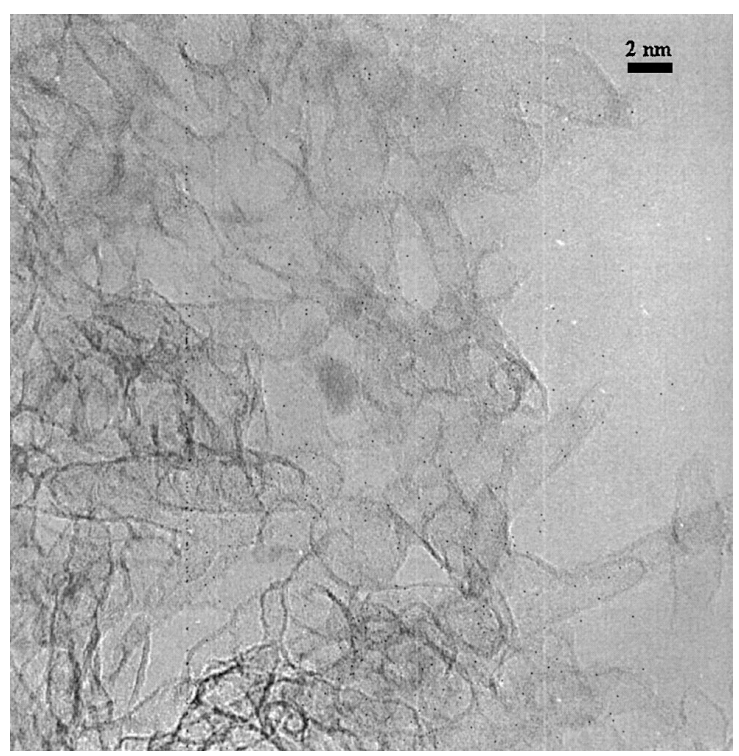

Fig. 4. The TEM image of the carbon tubules after a purification in an acid solution. 
solutions of equal amount of $\mathrm{HNO}_{3}(32.5 \mathrm{wt} . \%)$ and $\mathrm{H}_{2} \mathrm{SO}_{4}(48.5$ wt.\%)) reveals that both large catalyst particles, which are located outside the carbon tubule agglomerates, and the catalyst particles, which are located inside the carbon tubules, were removed by this treatment. The sizes and the microstructure of the carbon tubules, however, remained the same.

In contrast to polyethylene, the yield of carbonized solid products was significantly higher from the thermal decomposition of the polyvinyl alcohol. In Ref. [13], it was shown that the decomposition of polyvinyl alcohol without catalyst at $600^{\circ} \mathrm{C}$ (the sample PVA1) led to the formation of amorphous carbon. The catalytic decomposition of polyvinyl alcohol in the presence of the catalyst at $600^{\circ} \mathrm{C}$ (the sample PVA-
$\mathrm{Fe} 2)$ led to the formation of amorphous carbon and graphite (traces). According to XRD data (data not shown), the amorphous carbon and traces of graphite were detected in the sample PVA2, whereas iron carbide and graphite phases were seen in the sample PVA-Fe2.

The TEM image of the sample PVA-Fe2 (Fig. 5a) reveals the formation of three different carbon nanostructures, i.e. (a) multi-walled shells covered spherical catalyst particles, (b) cockle-shelled filaments, and (c) multi-walled graphite nanotubes. The multi-walled graphite shells completely isolate catalyst particles from their surrounding medium. Graphite nanofilaments, consisting of cockle-shells enclosed one into another, were formed in the bulk of the sample in the temperature range of $600-750^{\circ} \mathrm{C}$ (a)

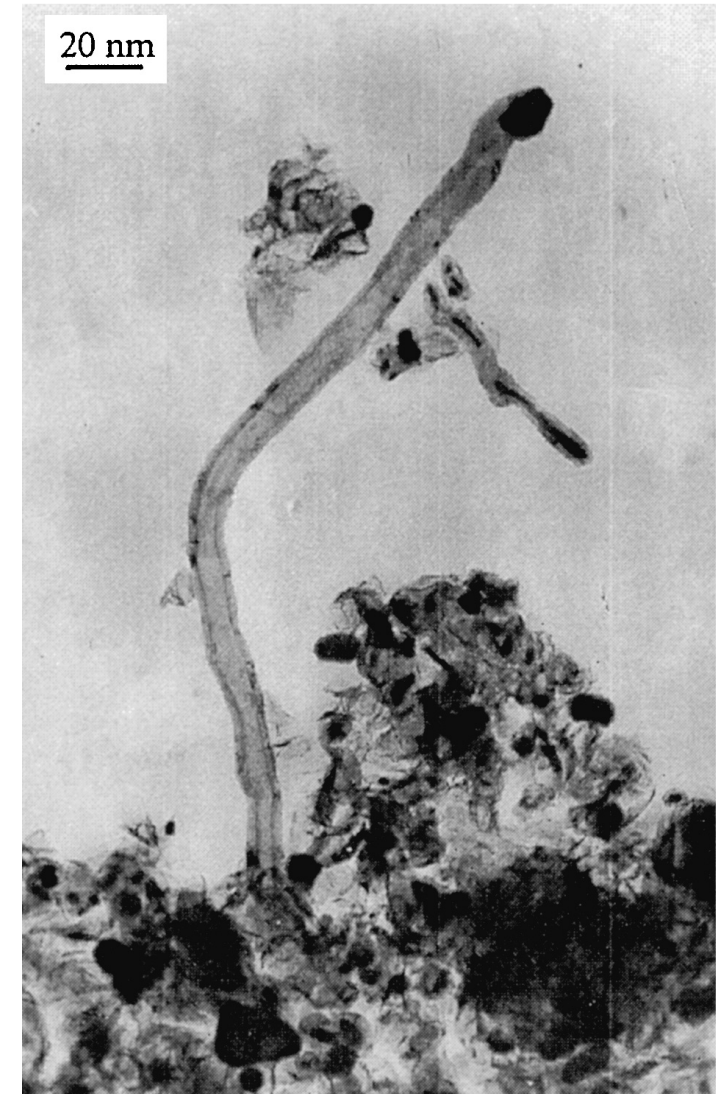

(b)

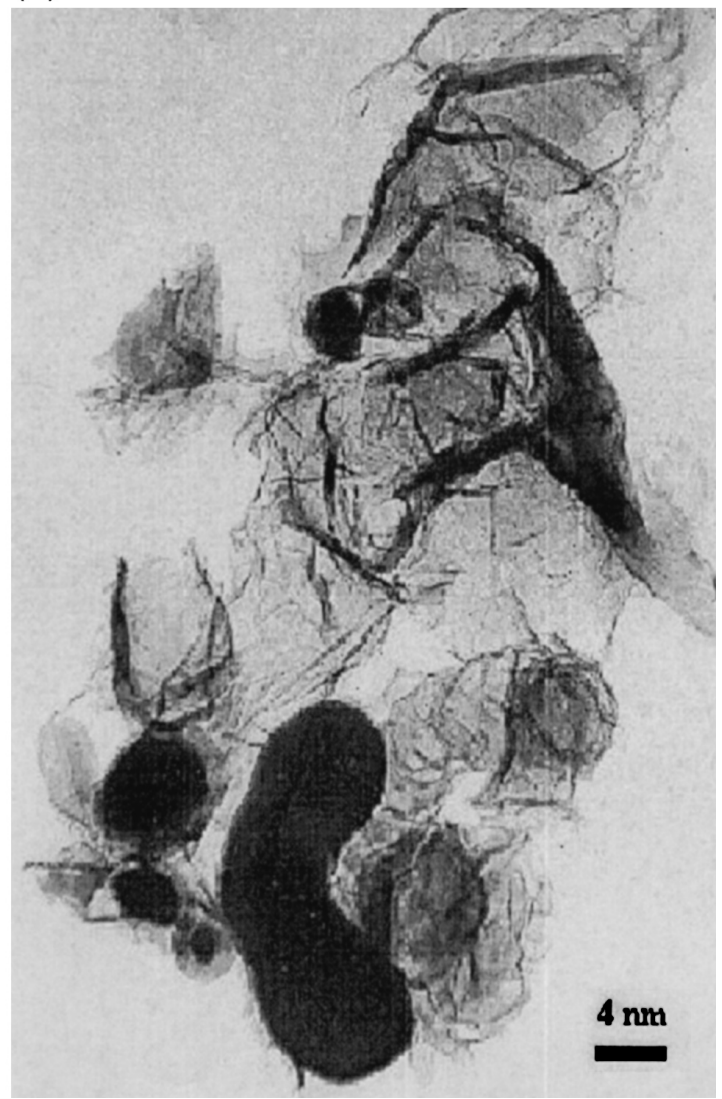

Fig. 5. The TEM image of (a) multi-walled graphite nanotube; (b) cocle-shelled filament obtained from the polyvinyl alcohol as the carbon precursor incorporating catalyst particles heated to $750^{\circ} \mathrm{C}$ in a nitrogen flow. 


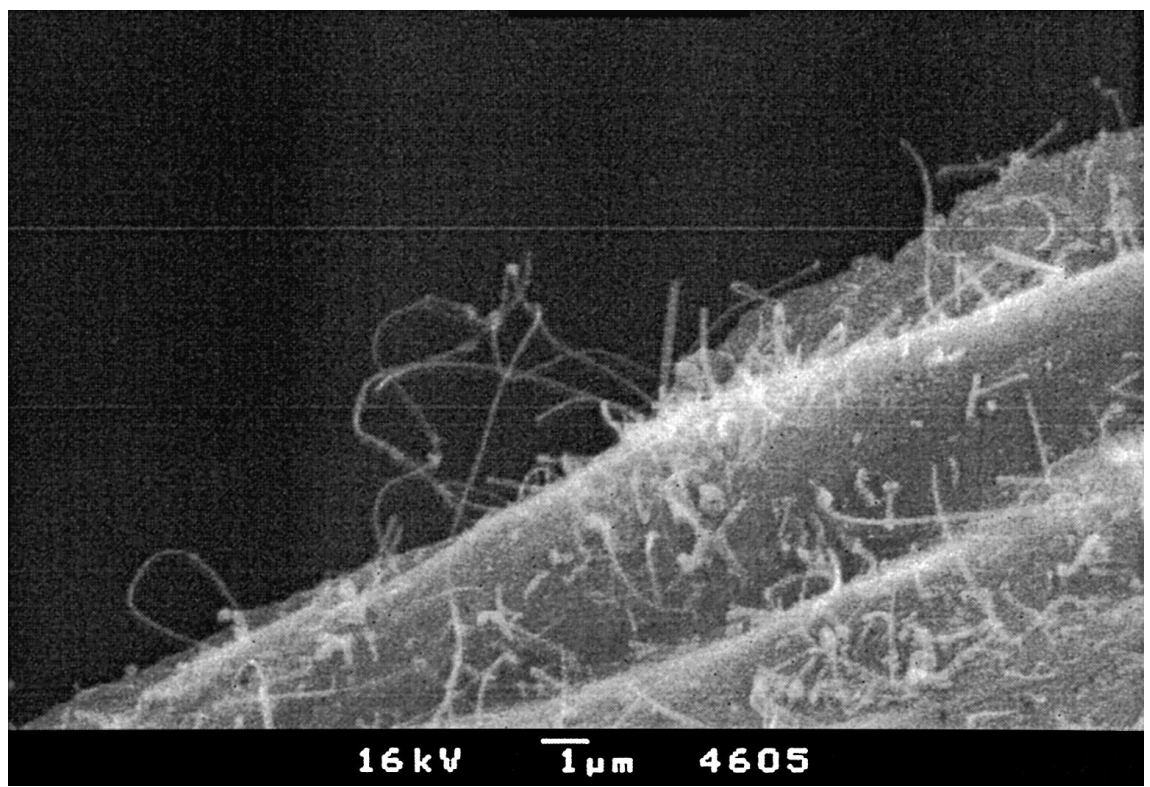

Fig. 6. The scanning electron micrograph of the sample of the polyvinyl alcohol incorporating catalyst particles heated to $750^{\circ} \mathrm{C}$ in a nitrogen flow.

due to the participation of catalyst particles located on the tips of filaments (Fig. 5b). According to TEM data (Fig. 5a), the growth of multi-walled nanotubes occurred from the ends of the cockle-shelled filaments. As a rule, the catalyst particles are located at the ends of graphite nanotubes and can also be observed within the channels of nanotubes. The mean outer diameter of the nanotubes is ca. $40 \mathrm{~nm}$ and mean length is ca. $200 \mathrm{~nm}$. The characteristic dimension of the catalyst particles of rounded shapes is about $40 \mathrm{~nm}$. The SEM image reveals that the nanotubes grew in the pore void of the sample (Fig. 6).

The IR and Raman spectroscopy data prove the presence of amorphous carbon and graphite in the samples PVA-Fe1 and PVA-Fe2 (Fig. 7a and $b$ ). Thus, for the sample PVA Fe1, the main stretching modes in the Raman spectrum of Fig. $7 \mathrm{~b}$ (1) correspond to the phonon spectrum of amorphous carbon and graphite (the mode at ca. $1350 \mathrm{~cm}^{-1}$ (D)) [18]. For the sample PVA-Fe2 (Fig. 7b (2)), the mode which appears at 1580 $\mathrm{cm}^{-1}(\mathrm{G})$ corresponds to graphite [18], the mode at $1333 \mathrm{~cm}^{-1}$ (D) to carbon nanotubes [19], and
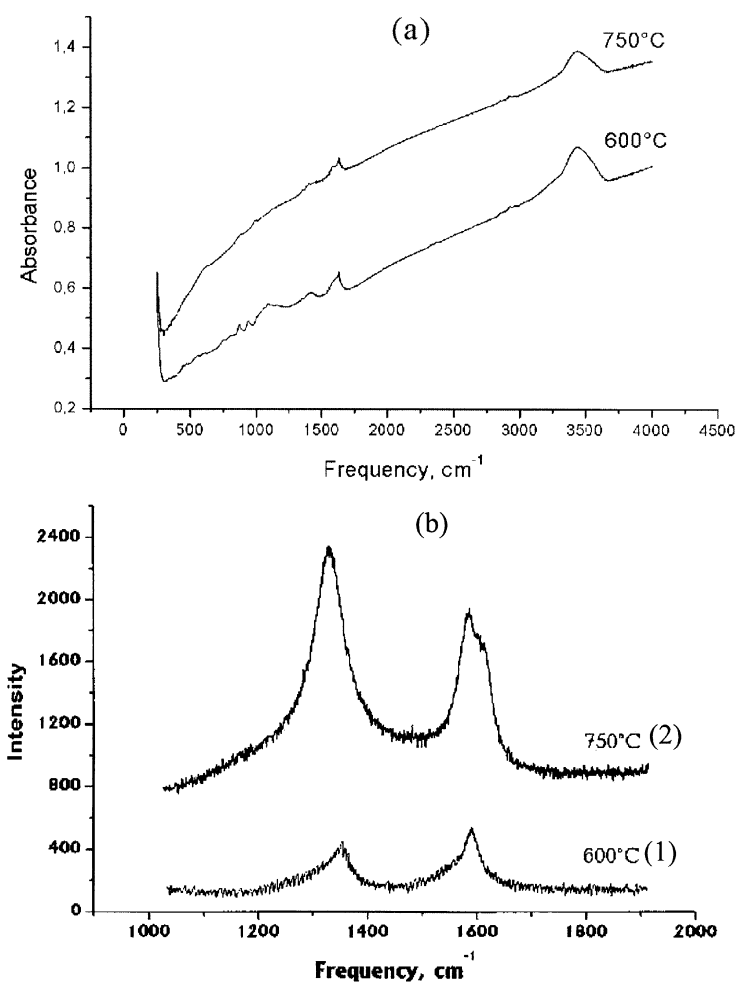

Fig. 7. The IR (a) and the Raman spectra (b) of the samples of polyvinyl alcohol incorporating catalyst particles heated to $600^{\circ} \mathrm{C}$ and $750^{\circ} \mathrm{C}$. 
the shoulder at $1600-1620 \mathrm{~cm}^{-1}\left(\mathrm{G}^{\prime}\right)$ to amorphous carbon.

The following mechanism for the formation of cockle-shelled filaments and multi-walled graphite nanotubes is suggested for the collected data. Iron metal produced from iron hydroxide accumulates carbon on its surface with the formation of $\mathrm{Fe}-\mathrm{C}$ intermediates. The $\mathrm{Fe}-\mathrm{C}$ intermediates transform into a liquid-like state and start to move while forming cockle-shelled filaments within the bulk of the amorphous carbon matrix. This cockle-shelled filament formation transports the catalyst particles to the matrix surface. The subsequent formation of multiwalled graphite nanotubes occurs in the pore voids of the matrix. Nanotubes may break apart from the cockle shells, and the catalyst particles remain attached to the ends of nanotubes.

\section{Conclusions}

In summary, a catalytic synthesis route to the different species of carbon nanotubes was presented starting from different carbon precursors in the presence of an iron catalyst uniformly distributed in the bulk polymer via heating to $750^{\circ} \mathrm{C}$ under a nitrogen flow. The catalytic synthesis of carbon nanotubes involves the catalytic decomposition of the polymer precursor to amorphous carbon and the simultaneous reduction of the iron hydroxide, used as the catalyst precursor, to iron metal at $600^{\circ} \mathrm{C}$ and $750^{\circ} \mathrm{C}$. In the case of the polyethylene carbon precursor, thin-walled carbon nanotubes are formed, whereas, cockle-shelled filaments and multiwalled graphite nanotubes are formed from the polyvinyl alcohol as a carbon precursor under the same conditions. The catalytic synthesis of carbon nanostructures via graphitization of amorphous carbon obtained from polymer precursors demonstrates a new route to produce different types of carbon nanostructures.

\section{References}

[1] H. Kroto, J. Heath, S. O’Brien, R. Curl, R. Smalley, Nature 318 (1985) 162.

[2] S. Iijima, Nature 354 (1991) 56.

[3] T. Fujimoto, A. Fukuoka, S. Iijima, M. Ichikawa, J. Phys. Chem. 97 (1993) 279.

[4] J. Bernholc, C. Roland, B.I. Yakobson, Curr. Opin. Solid State Mater. Sci. 2 (1997) 706.

[5] S. Iijima, T. Iishihashi, Nature 363 (1993) 603.

[6] Y. Saito, Carbon 33 (1995) 979.

[7] V. Ivanov, A. Fonseca, J.B. Nagy, A.A. Lucas, P. Lambin, D. Bernaerts, X.B. Zhang, Carbon 33 (1995) 1727.

[8] N.A. Kiselev, J. Sloan, D.N. Zakharov, E.F. Kukovitskii, Carbon 36 (1998) 1149.

[9] H. Marsh, A.B. Warburton, J. Appl. Chem. 20 (1970) 133.

[10] F.J. Derbishire, A.E.B. Presland, Carbon 13 (1975) 111.

[11] O.P. Krivoruchko, V.I. Zaikovskii, K.I. Zamaraev, Dokl. Akad. Nauk (Russia) 329 (1993) 744.

[12] O.P. Krivoruchko, V.I. Zaikovskii, Mendeleev Commun. 3 (1998) 97.

[13] N.I. Maksimova, O.P. Krivoruchko, Chem. Eng. Sci. 54 (1999) 4351.

[14] S.L. Madorsky, in: Thermal degradation of organic polymers, Wiley, New York, 1964, p. 231.

[15] N.I. Maksimova, O.P. Krivoruchko, A.L. Chuvilin, L.M. Plyasova, Carbon 37 (1999) 1657-1661.

[16] P. Godelle, Chem. Phys. Carbon 4 (1976) 137.

[17] V.N. Parmon, Catal. Lett. 42 (1996) 195.

[18] P.V. Huong, Diamond Diamond-Relat. Mater. 1 (1991) 33.

[19] P.V. Huong, R. Ajayan, R. Cavagnat, Phys. Rev. B 51 (1995) 10048. 